\title{
Introduction to Online Learning Volume 24, Issue 1
}

\author{
Peter Shea \\ University at Albany, SUNY \\ Editor of Online Learning
}

Our first issue of 2020 contains 14 articles in three sections. These papers are included in sections on student and faculty issues and concerns, as well as a collection of other empirical studies investigating online learning environments from a variety of theoretical and methodological approaches.

The first section includes four papers on faculty, professional development, and online teaching. This section begins with "Examining How Online Professional Development Impacts Teachers' Beliefs about Teaching Statistics" by Hollylynne Lee and Gemma Mojica of North Carolina State University and Jennifer Lovett of Middle Tennessee State University. In this study the authors are concerned with improving the teaching of statistics through online professional development provided through a Massive Open Online Course (MOOC). The data is drawn from 412 participants in the MOOC who identified themselves as classroom teachers. The central questions of the study center on identifying elements of the MOOC that trigger critical reflection and evidence that engaging in the MOOC influenced teachers' beliefs, perspectives, and practices in teaching statistics. The paper identifies aspects of the MOOC that hold promise in promoting positive change in teacher beliefs and practices.

The second paper in this section is "Facilitation Matters: Instructor Perception of Helpfulness of Facilitation Strategies in Online Courses" by Florence Martin, Chuang Wang, and Ayesha Sadaf of the University of North Carolina, Charlotte. In an earlier study some of these authors found online instructors' roles can be categorized as facilitator, course designer, course manager, subject matter expert, and mentor. This paper seeks to flesh out the role of facilitator by first reviewing the literature on facilitation and then presenting a result of a faculty survey. The results, from 100 online instructors, identify what they deem the most and least helpful facilitation strategies that were identified in the literature.

The next paper is "Social Media Learning Activities (SMLA): Implications for Design" by Ghania Zgheib of the University of Balamand, Lebanon, and Nada Dabbagh of George Mason University. There can be little doubt that our students are very engaged in social media use and that many instructors are exploring its educational applications. The author of this paper review promising research in this area and conclude that there is consensus on the benefits of social media use for learning. However, we need to better understand principles that can effectively guide SMLA design going forward. The paper investigates the types of learning activities designed through social media, the knowledge and cognitive processes they promote, and strategies experienced faculty use to design SMLAs.

The fourth paper in this issue is "Shifting Teaching and Learning in Online Learning Spaces: An Investigation of a Faculty Online Teaching and Learning Initiative" by Jayson Richardson and John Eric Lingat of the University of Kentucky, Ericka Hollis of Regis College, and Mikah Pritchard of Eastern Kentucky University. This study utilizes the Diffusion of 
Innovation (DoI) theory to interpret pedagogical changes that occurred as a result of professional development activities and a subsequent year-long faculty learning community and how perceptions of the diffusion of innovations characteristics influence the level of adoption of online/blended teaching. Results of the study indicate that participants most frequently mentioned experiences that refer to DOI components reflecting relative advantage, compatibility, and trialability.

The next section of this issue contains five papers broadly related to students, community, and online learning. This first of these is "From Discussion Forums to eMeetings: Integrating High Touch Strategies to Increase Student Engagement, Academic Performance, and Retention in Large Online Courses" by Glenda Gay of The University of the West Indies at Cave Hill and Kristen Betts of Drexel University. This paper utilizes an action research approach to examine strategies to address issues that can arise in larger format online courses: student disengagement, poor performance, and subsequent dropout. Based on data collected from more than 3,300 students over a six-year period, results indicate the use of the new eMeeting format integrating online high touch strategies, are correlated with significant increases in student engagement and academic performance. Additionally a comparison between the pre- and postintegration data revealed decreases in attrition, and higher scores on the standardized final exam. Course evaluations after the introduction of these strategies also reflect increased student satisfaction with the course. These approaches appear very promising and deserver further study.

The second paper in this section is "Postgraduate Online Teaching in Healthcare: An Analysis of Student Perspectives" by Cuisle Forde and Silvia Gallagher of Trinity College, Dublin. The goal of this study was to understand student perspectives in online health science courses. The main research questions explored in this study related to expectation and concerns healthcare students have before they start a postgraduate online course and their perceptions and experiences during and after the course. The authors to develop a set of recommendations for online educators that can serve as a guide for online course development and facilitation for students in the healthcare field.

The next paper is "Student Preferences for Learning Resources in a Land-based Postgraduate Online Degree Program" by Duncan Royd Slater of Myerscough College, Lancashire and Richard Davies of the University of Central Lancashire. This paper focuses on a newly emerging area of online study: specialized graduate education. Options for providing learning resources for such programs are myriad and understanding which resources and modes of delivery (e.g., text, audio, and video) are deemed useful is an important component of ensuring effectiveness. The study identified three resources currently used in the program that were significantly more favored than the others: online lectures, academic papers, and tutor's viewpoints. Other resources were rated lower. A number of responses showed there was a clear focus on relevant content over medium of delivery. This study demonstrates the importance of context in making decisions regarding the selection of resources for online learning.

The fourth paper in this section is "Factors Influencing Programming Expertise in a Webbased E-learning Paradigm" by Wajid Rafique, Wanchun Dou, and Khurshid Ahmed of Nanjing University and Khalid Hussain of East China University of Science and Technology. This study investigates the challenges of teaching computer programming in an online environment through the lens of the Technology Acceptance Model (TAM). Using data from 460 seniors in an undergraduate computer science program the authors validate and extend the TAM for students enrolled in computer programming. They conclude that teaching practices, intrinsic factors, 
perceived usefulness, efficacy problems, and learning intentions are key factors contributing toward programming expertise development in online learning environments. Much more detail is included in the full paper.

The next paper in this section is "The Development and Validation of the Distance Doctoral Program Integration Scale" by Joseph Holmes and Amanda Rockinson-Szapkiw of the University of Memphis. This paper seeks to provide insight into the issue of low online doctoral student persistence. The goal of this study is to close a gap in our understanding of academic integration and social integration of doctoral students enrolled in distance education programs. Specifically, the authors want to identify the underlying factors that explain integration of distance doctoral students. Based on a review of the literature they propose an instrument to measure integration of doctoral students and develop validity and reliability metrics for this instrument. This paper will be of interest to departments considering how to design new online doctoral programs that will engage students.

The final paper in this section is "Purposeful Interpersonal Interaction: What is it and How is it Measured?" by Scott Mehall of Carlow University. While we can all agree that interaction is an important contributor to learning in online settings, we can also acknowledge that not all interaction promotes learning or processes that contribute to learning. This paper provides an indepth investigation of the nature of productive interpersonal interaction to promote online learning. The author outlines a framework for purposeful interpersonal interaction characterized by three components: instructional, social, and support. These forms of interaction have been associated with either processes that support learning (a sense of community) or learning itself. The study details the conditions for creating these forms of productive interaction.

The final section of this issue contains four additional empirical studies on a variety of topics. The first paper is "The Role of an Interactive Visual Learning Tool and its Personalizability in Online Learning: Flow Experience" by Young Ha of California State University, Long Beach and Hyunjoo Im of the University of Minnesota, Twin Cities. Flow theory suggests that interactive visual learning tools have a high potential to engage students in learning processes and the effect is greater when the students' ability is close to the task difficulty level. This study tests these hypotheses with two experiments. The first experiment examines the effect of online interactivity on student learning process that manifest as flow experiences. The second experiment investigates whether students' learning experience is enhanced when students are able to match their skill level with the task difficulty through personalization options. Among other findings, the results demonstrate the important role of dynamic, real-time interactivity in improving students' learning by reducing awareness of physical surroundings and increasing flow states.

The next article in this section is "Using Structured Pair Activities in a Distributed Online Breakout Room" by Jeffrey Saltz and Robert Heckman of Syracuse University. Benefits of classroom-based collaborative learning when using breakout rooms include deeper learning, better grades, longer retention of information, greater communication and teamwork skills, and a better understanding of the professional environment in which students will work. How to structure breakout sessions in synchronous online environments is less well documented, especially for coursework in data science. This exploratory study seeks to close that gap by investigating a promising approach: structured pair activities, specifically using a strategy called "paired programming." In pair programming one member of the pair types at the keyboard while the other reviews each line as it is typed, checking for errors and thinking about the overall design. The paper provides observations of structured and unstructured student behaviors in online 
synchronous breakout rooms to highlight how the approach improves collaborative learning processes and outcomes.

The third paper in this section is "The Validity and Instructional Value of a Rubric for Evaluating Online Course Quality: An Empirical Study" by Ji Eun Lee and Mimi Recker of Utah State University, and Min Yuan of the University of Utah. Rubrics designed to assess the quality of online course design are commonly used in higher education institutions, but few have been empirically tested for reliability or validity. Even fewer have been assessed for their influence on promoting productive online interactions or actual student outcomes (e.g., grades). This paper seeks to address that issue by providing validity and reliability measures for an online course quality rubric and tying those metrics to learner outcomes (course passing rates). Using data from 121 online courses enrolling 5,240 students, an analysis demonstrates that only rubric items related to learner engagement and interaction have positive effects on online interactions, while only student-content interaction positively influence course passing rates. This paper will be on interest to faculty and instructional designers seeking to improve the quality of online coursework.

The next paper is "A Dramaturgical Examination of Online University Student Practices in a Second Year Psychology Class" by Dawn Marie Gilmore of the Royal Melbourne Institute of Technology, Australia. This study adopts a framework based on Erving Goffman's classic sociological theory which posits metaphors for the presentation of self as being either on the front stage or the backstage. If the Learning Management System (LMS) is conceived as the front stage, then other environments that students use to prepare for their performance in the LMS combine to form the students' backstage learning environment. This study analyzes what students do beyond the LMS and how social media spaces (especially Facebook) preferred by students support social learning and enhance the student experience. In part the paper concludes that some students avoid the front stage discussion board because the audience is too slow, too harsh, and too formal. The backstage online audience in social media solved these drawbacks of the front stage, which made it a more attractive option for learning.

We invite you to read and share this issue with colleagues and to consider submitting your own original work to Online Learning. 